Umfang seines Rechts im Ungewissen ist, er sich die zur Vorbereitung und Durchsetzung seines Anspruchs notwendigen Auskünfte nicht in zumutbarer Weise selbst beschaffen kann und der Verpflichtete unschwer, d.h. ohne unbillig belastet zu sein, die zur Beseitigung dieser Ungewissheit erforderlichen Auskünfte zu geben vermag (BGH, NJW 2015, 1525, Rdnr. 7 m. w. N.).

Jedenfalls ist die von der Kl. begehrte Auskunft, soweit sie hier dem Grunde nach noch in Rede steht, der Bekl. also nicht unmöglich ist (vgl. oben 1.), für die Bekl., nicht unschwer zu erteilen und ihr daher nicht zuzumuten. Eine Auskunft ist dann nicht unschwer zu erteilen, wenn der Anspruchspflichtige unbillig belastet wird, was jeweils unter Abwägung aller Umstände des Einzelfalles zu beurteilen ist (BGH, MW 2007, 1806). Dabei ist hier entscheidend, dass es (nur noch) um solche Unterlagen geht, die nicht die Behandlung der Kl. oder sonst die Vertragsbeziehung unmittelbar betreffen, sondern allgemeine interne Abläufe und Verhältnisse dokumentieren. Es ist der Bekl. als Krankenhausbetreiberin in Anbetracht des hieraus folgenden Aufwands nicht zuzumuten, jedem Patienten umfassende Einsicht in (potentiell sämtliche) allgemeine Unterlagen zu gewähren, um Anhaltspunkte für eine mögliche Haftung zu finden. Würde man dies anders sehen und einen Auskunftsanspruch aus $\$ 242$ BGB annehmen, liefen die oben dargestellten Wertungen der spezielleren Auskunftsansprüche im Ergebnis leer. [...]

https://doi.org/10.1007/s00350-018-4915-y

\section{Anmerkung zu OLG Karlsruhe, Urt. v. 3.8.2017 - 7 U 202/16 (LG Mannheim)}

\section{Albrecht Wienke}

Das OLG stellt klar, dass allgemeine Dokumentationen, welche die interne Organisation eines Krankenhauses betreffen, nicht zu den Behandlungsunterlagen im Sinne des $\$ 630 \mathrm{f} \mathrm{BGB} \mathrm{zu} \mathrm{zählen} \mathrm{sind.} \mathrm{Entsprechend} \mathrm{besteht} \mathrm{kein}$ Recht der Patienten zur Einsichtnahme in eben solche Dokumente. Entscheidend für das Recht zur Einsichtnahme ist, dass ein tatsächlicher Bezug zur Behandlung des konkreten Patienten bestehen muss, was bei allgemeinen Protokollen über die Sterilisation von OP-Bestecken, die nicht einem bestimmten Patienten zugeordnet werden können, nicht der Fall ist. Anders wäre der Fall zu beurteilen gewesen, wenn über die konkret bei der Patientin verwendeten OP-Instrumente ein Sterilisationsprotokoll vorgelegen hätte. Da ein solches jedoch unstreitig nicht im Krankenhaus existierte, ging auch die darauf gerichtete Herausgabeklage ins Leere.

Eine Absage erteilte der Senat der gelegentlich anzutreffenden Meinung, dass das Führen der Krankenakte auch der Durchsetzung von späteren Schadensersatzansprüchen dienen müsse. Das OLG stellte insofern klar, dass eine Dokumentation, die aus medizinischen Gründen nicht geboten sei, auch aus Rechtsgründen nicht geboten sei. Der Anspruch aus $\$ 630 \mathrm{~g}$ BGB umfasst daher nur die vollständige Patientenakte, zu deren Führen der Behandelnde gesetzlich verpflichtet ist. Dazu gehören „neben den medizinischen, objektivierbaren Befunden und Berichten über Behandlungsmaßnahmen wie Operationen und Medikation auch die Schilderung subjektiver Wahrnehmung und persönlicher Eindrücke des Behandelnden (...). "Für den Umfang dessen, was $\mathrm{zu}$ dokumentieren ist, gilt $\$ 630 \mathrm{f}$ BGB. Danach sind die

Rechtsanwalt Dr. iur. Albrecht Wienke, Fachanwalt für Medizinrecht, Wienke \& Becker Rechtsanwälte,

Sachsenring 6, 50677 Köln, Deutschland für die Behandlung wesentlichen Maßnahmen und deren Ergebnisse, die aus der fachlichen Sicht des Behandelnden für die Sicherstellung der derzeitigen oder einer künftigen Behandlung wesentlich sind oder sein können, zu dokumentieren. Dokumentationen über die innere Organisation eines Krankenhauses, die den gesamten Krankenhausbetrieb betreffen, gehören daher nicht dazu.

Der Senat nimmt damit eine wichtige Konkretisierung im Hinblick auf den Umfang des Einsichtsrechts der Patienten vor. Allerdings darf daraus nicht generell geschlussfolgert werden, dass solche Dokumente im Gerichtsverfahren nicht vorgelegt werden müssen. $\mathrm{Zu}$ berücksichtigen ist hier, dass es sich um eine reine Herausgabeklage handelte. Im Arzthaftungsprozess hingegen wird es vielfach erforderlich sein, schon im eigenen Interessen des Krankenhauses entsprechende Dokumente vorzulegen, um die erhobenen Vorwürfe des Patienten zu entkräften. Der BGH hat insoweit mit Beschluss v. 16.8.2016 ${ }^{1}$ klargestellt, dass es bei Fehlern im Bereich des vollbeherrschbaren Risikos zu einer Umkehr der Beweislast kommt. Erforderlich ist allerdings hierfür, dass der Patient einen konkreten Fehler des Krankenhauses nachweist, was allein beim Auftreten einer Infektion im Zusammenhang mit einer Krankenhausbehandlung nicht angenommen werden kann. Die Hürde für einen entsprechenden Nachweis liegt daher entsprechend hoch.

Allerdings hat der $\mathrm{BGH}$ in dieser Entscheidung auch klargestellt, dass für den Fall, dass der Patient Hinweise für Fehler bei der Krankenhaushygiene nachweist, das Krankenhaus im Rahmen einer sogenannten sekundären Darlegungslast erklären und belegen muss, dass die entsprechenden Hygienevorschriften eingehalten worden sind. In einem solchen Fall ist daher die Vorlage entsprechender interner Hygieneprotokolle etc. von großer Bedeutung.

Wann vom Nachweis eines Hinweises auf Fehler gesprochen werden kann, ist von Fall zu Fall zu beurteilen. Es ist daher nicht ausgeschlossen, dass Gerichte einen solchen Hinweis auch - wie im vorliegenden Fall - in der Pressemitteilung über Hygienedefizite in einem Krankenhaus sehen würden.

Auch wenn kein unmittelbarer Anspruch der Patienten auf Einsichtnahme in interne Hygieneprotokolle eines Krankenhauses besteht, sollte daher dennoch großer Wert auf die Beachtung und entsprechende Dokumentation gelegt werden, um in einem möglichen späteren Schadensersatzprozess keine prozessualen Nachteile zu erleiden.

1) BGH, Beschl. v. 16.8.2016 - VI ZR 634/15 -, GesR 2016, $701 \mathrm{ff}$

\section{Zur Definition des Begriffs „Praxisklinik“}

$\S 115$ Abs. 2 S. 1 Nr. 1 SGB V, §§ 8 Abs. 1, 3 Nr. 3; 3 und 5 Abs. 1 S. 2 Nr. 1 und 3 UWG.

Die Werbung eines Zahnarztes mit dem Begriff ,Die Praxisklinik“" ist zulässig, auch wenn dort kein stationärer Aufenthalt möglich ist.

LG Essen, Urt. v. 8. 11.2017 - 44 O 21/17

Problemstellung: Verbände zur Förderung gewerblicher Interessen und Verbraucherschutzorganisationen im Sinne des $\$ 8$ Abs. 3 UWG sind neben jedem Mitbe-

Eingesandt von Prof. Dr. iur. Martin Stellpflug, Berlin;

bearbeitet von Dr. iur. Christoph Rosset, Schwerpunkt Arztrecht, Rechtsanwälte Rosset, Merz \& Partner PartGmbB, Schwarzwaldstr. 1, 79117 Freiburg, Deutschland 